\title{
Sistema de biofeedback para rehabilitación de marcha asistida por un exoesqueleto
}

\author{
John Jairo Villarejo Mayor ${ }^{1, \psi}$, Nicolás Jacobo Valencia Jiménez ${ }^{1}$, Gloria Patricia Arango Hoyos², \\ Eduardo Francisco Caicedo Bravo ${ }^{1}$ \\ ${ }^{1}$ Grupo de Percepción y Sistemas Inteligentes, Universidad del Valle, Cali, Colombia \\ ${ }^{2}$ Grupo de Investigación Sinergia, Universidad del Valle, Cali, Colombia
}

Recibido 8 de julio de 2015. Aceptado 18 de enero de 2017

\begin{abstract}
Resumen-Los robots proporcionan nuevas formas de terapia para pacientes con desórdenes neurológicos. Las terapias de marcha asistidas con exoesqueletos pueden incrementar la duración y la intensidad de los entrenamientos para los pacientes y reducir el esfuerzo físico del terapeuta. Sin embargo, el uso de estos dispositivos para el entrenamiento de la marcha limita la interacción física entre el terapeuta y el paciente, en comparación con la terapia manual. Una apropiada realimentación de las funciones corporales y biomecánicas en la interacción con el sistema robótico facilita la evaluación del desempeño del paciente, motivándolo en el reaprendizaje de la marcha con resultados superiores. Este artículo presenta el diseño de una interfaz de usuario para un exoesqueleto de miembros inferiores para asistencia en la marcha y en terapias de rehabilitación. Se consideraron aspectos técnicos y clínicos para proporcionar ventajas del exoesqueleto durante las terapias, estableciendo una herramienta de apoyo para la configuración, monitoreo y registro de los parámetros involucrados. Se propuso un esquema de realimentación sensorial para el paciente acerca de la actividad muscular, la presión ejercida en diferentes puntos de los pies y algunas variables biomecánicas. Finalmente, se valida la herramienta con sujetos sanos por medio de un test de usabilidad propuesto.
\end{abstract}

Palabras clave-Electromiografía de superficie sEMG, Exoesqueleto de miembros inferiores, Interfaz de usuario, Rehabilitación de la marcha.

\section{Biofeedback System for Exoskeleton Assisted Gait Rehabilitation}

\footnotetext{
Abstract - The inclusion of robots in rehabilitation allow advantages for generate newer therapies in neurologic disorder patients. Assistive gait therapies using robots, like exoskeletons, allow increase the time and intensity training for patients while the strenuous labor of therapist is reduced. However, the physic interaction between therapist and patient in training with robots is limited, in relation to the traditional manual therapy. An appropriated feedback of biological and biomechanics functions in the
} 
robot interaction during training provides an easier performance evaluation of the patient for the therapist. Further, biofeedback gives a motivation to the patient and encourages him for gait relearning with higher effects than conventional. This paper presents a user interface design for a lower limb exoskeleton for human gait assistance in rehabilitation. Clinical and technical criteria for increasing the advantages of the exoskeleton in therapy were considered. A biofeedback scheme about muscle activity, plantar pressure and some biomechanics variables, for the patient is proposed. Finally, a validation for this tool with healthy subjects by a usability test was carried out.

Keywords - Gait rehabilitation, Lower limb exoskeleton, Superficial electromyography sEMG, User interface.

\section{SISTEMA DE BIOFEEDBACK PARA REABILITAÇÃO DE MARCHA ASSISTIDA POR UM EXOESQUELETO}

Resumo-A inclusão de robôs na reabilitação fornecem vantagens que promovem novas formas de terapia em pacientes com desordens neurológicas. Terapias de marcha assistidas por exoesqueletos permitem o aumento da duração e da intensidade dos exercícios com os pacientes, reduzindo o esforço físico dos terapeutas. Não entanto, o uso desses dispositivos para o treino da marcha limita a interação física entre o terapeuta e paciente, em comparação com a terapia manual. Uma apropriada realimentação das funções corporais e biomecânicas na interação com o sistema robótico facilita a avaliação do progresso do paciente, motiva e incentiva ao paciente na reaprendizagem da marcha gerando efeitos superiores aos convencionais. Neste artigo apresenta-se o desenho de uma interface de usuário para um exoesqueleto de membros inferiores para assistência na marcha e nas terapias de reabilitação. São considerados aspectos técnicos e clínicos para fornecer maiores vantagens do exoesqueleto durante as terapias, estabelecendo uma ferramenta de suporte para configuração, monitoramento e registro dos parâmetros envolvidos. Foi proposto um sistema de realimentação sensorial para o paciente sobre a atividade muscular, a pressão em diferentes pontos dos pés e algumas variáveis biomecânicas. Finalmente, é apresentada a ferramenta de validação para indivíduos saudáveis utilizando um teste de usabilidade proposto.

Palavras-chave-Eletromiografia de superfície sEMG, Exoesqueleto de membros inferiores, Interface do usuário, Reabilitação da marcha.

\section{INTRODUCCIÓN}

L as lesiones en la médula espinal y enfermedades cerebrovasculares pueden producir una reducción parcial o total de las capacidades motoras. Debido a la pérdida de tejidos nerviosos el cerebro es incapaz de controlar ciertos músculos, que aunque no están completamente dañados, inhiben sus funciones, haciendo necesario un programa de rehabilitación [1]. Estas lesiones pueden provocar pérdida del control motor, grados variables de debilidad muscular y trastornos de la sensibilidad.

Los procesos de rehabilitación física generalmente presentan importantes limitaciones, entre las que se cuen$\tan$ los tiempos reducidos para las sesiones de terapia y la imposibilidad para garantizar una práctica consistente de la actividad, aún con la presencia permanente del terapeuta. Se suma a la problemática expuesta, las limitaciones en los procesos de medición de la condición del paciente y su recuperación, que en la mayoría de los casos son de carácter subjetivo y no evalúan de forma consistente la evolución en el tratamiento. La problemática planteada abre un creciente interés por obtener mediciones objetivas, confiables y repetibles acerca de estos procedimientos, y por vincular ayudas técnicas que permitan aumentar los tiempos de práctica y ajustar la exigencia para la recuperación del paciente.
La ejercitación de las articulaciones juega un papel importante en la rehabilitación para restaurar las funciones musculares y el control motor, además de prevenir limitaciones en la movilidad articular. En pacientes con desórdenes neurológicos la rehabilitación debería incluir un reentrenamiento de la marcha en función de trayectorias deseadas de acuerdo a un programa específico. Actualmente se acepta que el tratamiento efectivo de las lesiones no consiste en terapias pasivas y de reposo, sino en el ejercicio y en el restablecimiento funcional [2]. El ejercicio activo ayuda a mantener la flexibilidad de las articulaciones evitando rigidez y limitaciones musculares, además permite trabajar directamente sobre la musculatura proporcionando estabilidad al paciente [3]. El soporte del peso y la marcha son las funciones más importantes de los miembros inferiores y su rehabilitación debería orientarse hacia la restauración de un alineamiento adecuado en posición bípeda y la restauración de la habilidad para caminar de manera estable y segura.

El aprendizaje motor es un proceso de adquisición de capacidades para realizar acciones con destreza, que resulta de la experiencia y la práctica y que produce cambios permanentes en el comportamiento [4]. Se ha logrado establecer también que el inicio temprano del entrenamiento y la práctica de tareas específicas favorecen el aprendizaje 
motor [5]. Algunos estudios han mostrado que el aumento de la intensidad en el entrenamiento podría conducir a mejores resultados [6-8]. Sin embargo, en la terapia manual las piernas del paciente son guiadas por el terapeuta quien controla y asegura los movimientos en trayectorias, lo que termina siendo una tarea ardua y poco consistente. En algunos casos, los terapeutas tienen que asistir la postura de la pierna extendiendo la cadera y la rodilla en contra del peso del paciente o flexionando la rodilla, incluso en contra de la espasticidad. El esfuerzo físico intenso de los terapeutas limita la duración de los entrenamientos, mientras que el paciente se beneficiaría con una mayor duración.

Se han desarrollado diversos sistemas robóticos para ofrecer soporte continuo para las piernas, brindando un patrón de marcha fisiológico repetido con una alta exactitud por periodos de tiempo prolongados en relación a la terapia manual [9-12]. La pérdida del contacto físico entre el paciente y el terapeuta representa una desventaja, pero puede ser superada con el apoyo de la tecnología. Por medio del contacto físico el terapeuta podía sentir la actividad del paciente y determinar subjetivamente sus habilidades, proporcionando una realimentación al paciente, tanto para determinar el entrenamiento adecuado, así como para ayudar a mejorar la motivación del paciente.

Se ha demostrado que la realimentación de la actividad del sistema de rehabilitación robótico podría substituir estas limitaciones, basado en que la información obtenida sobre el desempeño de la terapia permite mejorar los efectos del entrenamiento [13]. El término en inglés biofeedback ha sido ampliamente usado para definir el tipo de realimentación artificial de las variables biológicas medidas en la interacción humano-robot, que son transferidas sensorialmente al sistema biológico, en este caso el cuerpo humano [14].

Los movimientos del cuerpo humano normalmente son realimentados y controlados por sistemas aferentes tales como la propiocepción, fuerza o y sensores visuales, también conocidos como realimentación intrínseca [15]. Esta realimentación es generada por el propio movimiento, ya sea por la propiocepción o por la visualización de la parte del cuerpo en movimiento. Por el contrario, la realimentación extrínseca puede ser proporcionada por una fuente externa, como el terapeuta $[11,15]$.

Mantener a los pacientes informados sobre su progreso puede llevar a una mayor exigencia en el esfuerzo durante el entrenamiento. Esto hace que la realimentación pueda ayudar en la motivación y en la mejora de los resultados del entrenamiento [14]. Se requieren entonces de mediciones para comparar el estado actual respecto del deseado, que pueda además establecer la cantidad y la calidad de los movimientos realizados por el paciente.
El biofeedback ha sido aplicado en rehabilitación de marcha con pacientes con accidente cerebro-vascular y con lesiones en la médula espinal [16]. Registros de electromiografía (EMG) [17] y variables cinemáticas han sido procesados para presentar estímulos visuales, acústicos [18] o como combinación de ambas [19], así como también con métodos vibrotáctiles [20]. La aplicación de biofeedback en rehabilitación de lesiones neurológicas ayuda a mejorar las funciones motoras del paciente durante la marcha [21].

En la terapia manual, el terapeuta estima el nivel de asistencia necesaria para asegurar que le paciente mantenga un patrón de marcha fisiológico. Sin embargo, cuando se reduce el desempeño del movimiento este tipo de asistencia comúnmente se incrementa para obtener una mejora, llevando posiblemente a que el paciente camine con un esfuerzo inferior al de su capacidad máxima. Por tanto, la estimación de la capacidad máxima de locomoción del paciente y su realimentación podrían mejorar la calidad del entrenamiento de la marcha.

Los sensores de fuerza se pueden usar para medir el nivel de asistencia generada por el robot para el seguimiento de los patrones de marcha. Por otro lado, la electromiografía de superficie (sEMG) registra la actividad eléctrica generada por un grupo de fibras musculares relacionadas a determinada actividad. La sEMG captura información referente a los músculos que actúan en una actividad dinámica como la marcha, incluyendo un gradiente otros músculos cercanos a la región de captura.

La electromiografía superficial (EMGs) permite obtener una valoración del número de fibras musculares que son reclutadas para realizar un movimiento en particular, que finalmente refleja el nivel de esfuerzo físico realizado relacionado con la amplitud de la señal en el dominio temporal y la frecuencia de reclutamiento de estas fibras. Esto se relaciona directamente con el grado de contracción de los músculos durante un movimiento específico, haciendo posible determinar cuáles están siendo utilizados y cuándo se realizan compensaciones inadecuadas con otros [22].

Una de las técnicas más utilizadas en rehabilitación es el biofeedback electromiográfico, que detecta la actividad mioeléctrica mediante electrodos de superficie para generar información auditiva o visual útil al paciente para inhibir o aumentar determinada actividad muscular [2325]. Otra forma de realizar biofeedback se hace a partir de la información de una plataforma sensible a la presión durante la bipedestación, para reentrenar la simetría en el apoyo y el equilibrio (estático y dinámico); también la posición articular obtenida con un electrogoniómetro puede ser utilizada para este fin [26]. 
Algunos dispositivos robóticos son empleados para asistir la estimulación sensitivo-motora obteniendo mejoras significativas en la recuperación motora de los músculos reentrenados [27]. Un componente esencial de los dispositivos de asistencia robótica lo constituye la interfaz de usuario, que comunica la máquina con el hombre, permitiendo la interacción entre ellos y el monitoreo de señales del equipo y el paciente durante su utilización. Adicionalmente, se han utilizado programas informáticos interactivos con los cuales se le proporciona realimentación sensorial (biofeedback) al paciente mediante señales acústicas y visuales y se permite analizar y monitorear algunas funciones motoras [28]. Varios estudios han demostrado mejoras significativas en los procedimientos de rehabilitación tanto ortopédicos como neurológicos al incluir herramientas o dispositivos que permitan obtener valoraciones objetivas y llevar a cabo procedimientos más prolongados, repetibles y precisos.

Actualmente, importantes centros de rehabilitación incluyen el uso de aparatos robóticos para facilitar los procesos de rehabilitación, especialmente en situaciones en las que se requiere la práctica de acciones específicas con repetición constante y precisa. Este es el caso del entrenamiento de la marcha en pacientes con secuelas de Enfermedades Cerebrovasculares (ECV), Traumatismo Craneoencefálico (TCE) y Traumatismo Raquimedular (TRM) [12, 29-31].

Los trabajos de investigación que se están desarrollando con estos grupos de pacientes han demostrado que la asistencia con control robótico sumada a la terapia convencional aumenta la duración del entrenamiento, permite entrenar de manera temprana un patrón de marcha más fisiológico y simétrico y posibilita medir y documentar la participación del paciente; además facilita el trabajo para el equipo de rehabilitación, requiriendo menos personas para asistir a un solo paciente [1].

En la Universidad del Valle, Colombia, se han desarrollado algunos adelantos en cuanto a dispositivos para rehabilitación física. El primero de ellos, un exoesqueleto de rodilla para rehabilitación física, permite la transferencia de cargas y soporte actuado para entrenamientos activos y pasivos de flexo-extensión de rodilla. El dispositivo consta de un sistema de control electrónico con controlador, actuador y un conjunto de sensores adjuntos que se integran a módulo central, proporcionando una amplia variedad de información respecto a la cinemática y a la cinética del exoesqueleto [32].

El segundo dispositivo consiste en un exoesqueleto para rehabilitación asistida de pacientes con pérdida parcial o completa del movimiento de MMII, con soporte en el troco y con cadera y rodillas accionadas. Fue concebido para pacientes con TCE, ECV o TRM, que requieran asistencia para mantener el equilibrio y en tareas de rehabilitación de marcha y de movilidad de miembros inferiores.

En [33] se describe el desarrollo de una interfaz de usuario como herramienta de configuración, monitoreo y realimentación sensorial para el exoesqueleto de MMII mencionado anteriormente (Fig. 1). Se presenta un esquema general que compone la herramienta, su funcionalidad y se exponen las ventajas que representa la herramienta en el tratamiento con pacientes.

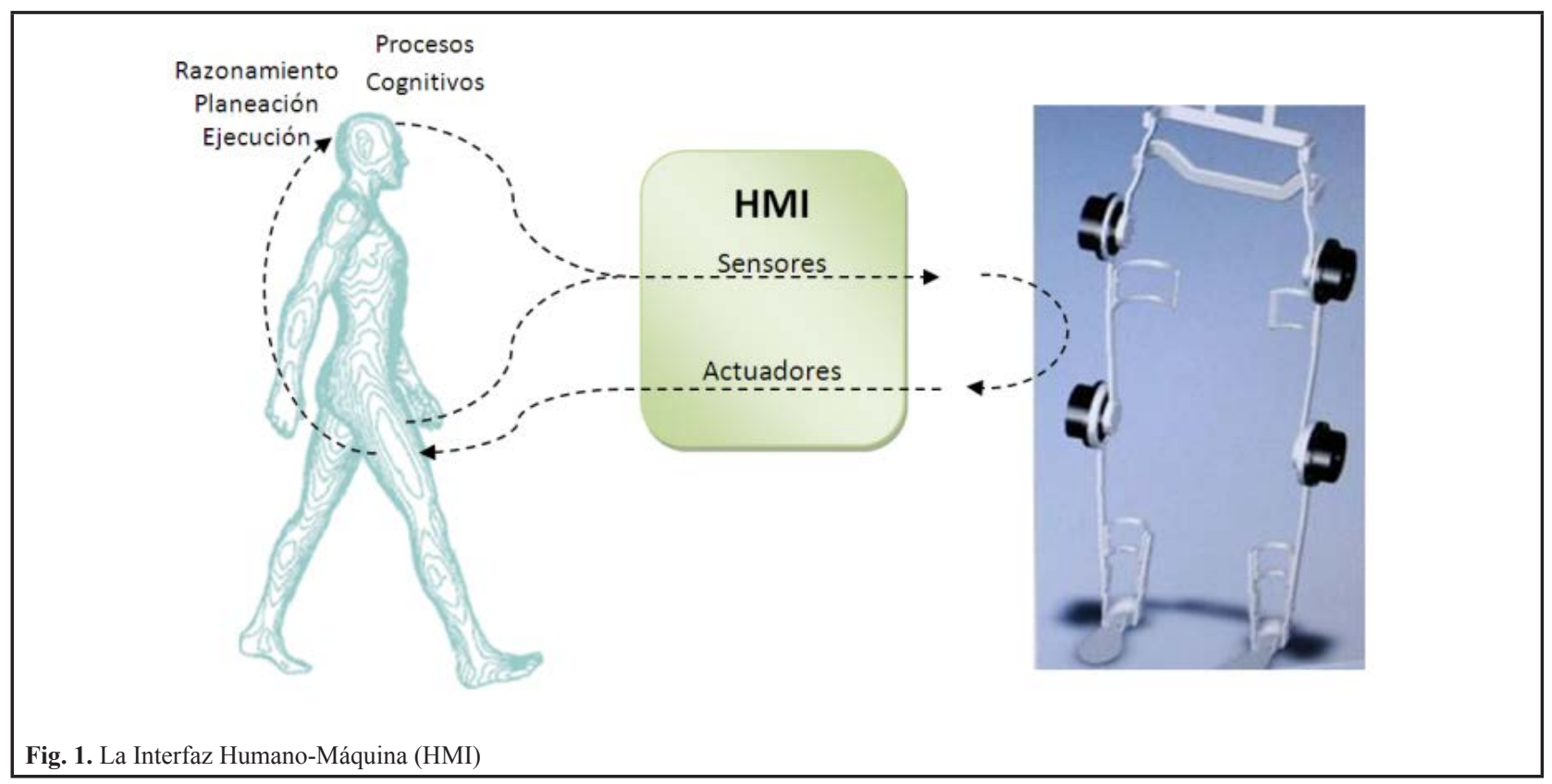




\section{Materiales y Métodos}

\subsection{Exoesqueleto}

El exoesqueleto para rehabilitación asistida de pacientes con pérdida parcial o completa del movimiento de MMII, cuenta con soporte en el tronco y accionamiento en cadera y rodillas (Fig. 2). El exoesqueleto cuenta con un sistema de control de equilibrio en bipedestación. Se compone de una estructura mecánica (órtesis), un sistema de actuación en cuatro articulaciones y un sistema de instrumentación que se encarga de controlar y medir el estado de variables como torque, velocidad, ángulo y aceleración en las articulaciones, presión en los pies y señales electromiográficas. Cuenta con sensores de ángulo de Efecto Hall; sensores de presión (Flexiforce-resistivos); sensores de aceleración lineal (triaxiales) y giróscopos (triaxiales); y un circuito de adecuación de señales electromiográficas de superficie sEMG de 4 canales por cada miembro inferior. Los actuadores y los instrumentos se conectan a nodos concentradores y de estos, a un procesador principal a través de una red de datos cableada con el protocolo SPI.

Los parámetros medidos en la estructura mecánica y las señales biológicas capturadas en el paciente son:
- Señales de sEMG

- Ángulo de las articulaciones de cadera, rodilla y tobillo

- Velocidad angular de rodilla

- Presión en cuatro puntos de la planta del pie

- Torque de los motores en las articulaciones de cadera y rodilla

\subsection{Interfaz de usuario}

De acuerdo al potencial del exoesqueleto y a las necesidades reunidas por los terapeutas, se tomaron en cuenta principalmente las tareas relacionadas con los programas de fortalecimiento muscular y neurorehabilitación. Para el entrenamiento en programas de fortalecimiento muscular se consideraron los ajustes de resistencia al movimiento activo-asistido en cada arco de movimiento. Por otra parte, en neurorehabilitación se pueden realizar una gran variedad de facilitaciones de tareas motoras, reeducación de patrones de marcha, acondicionamiento físico en general y optimización de la funcionalidad en actividades de la vida diaria y básicas cotidianas, como desplazamientos y traslados.

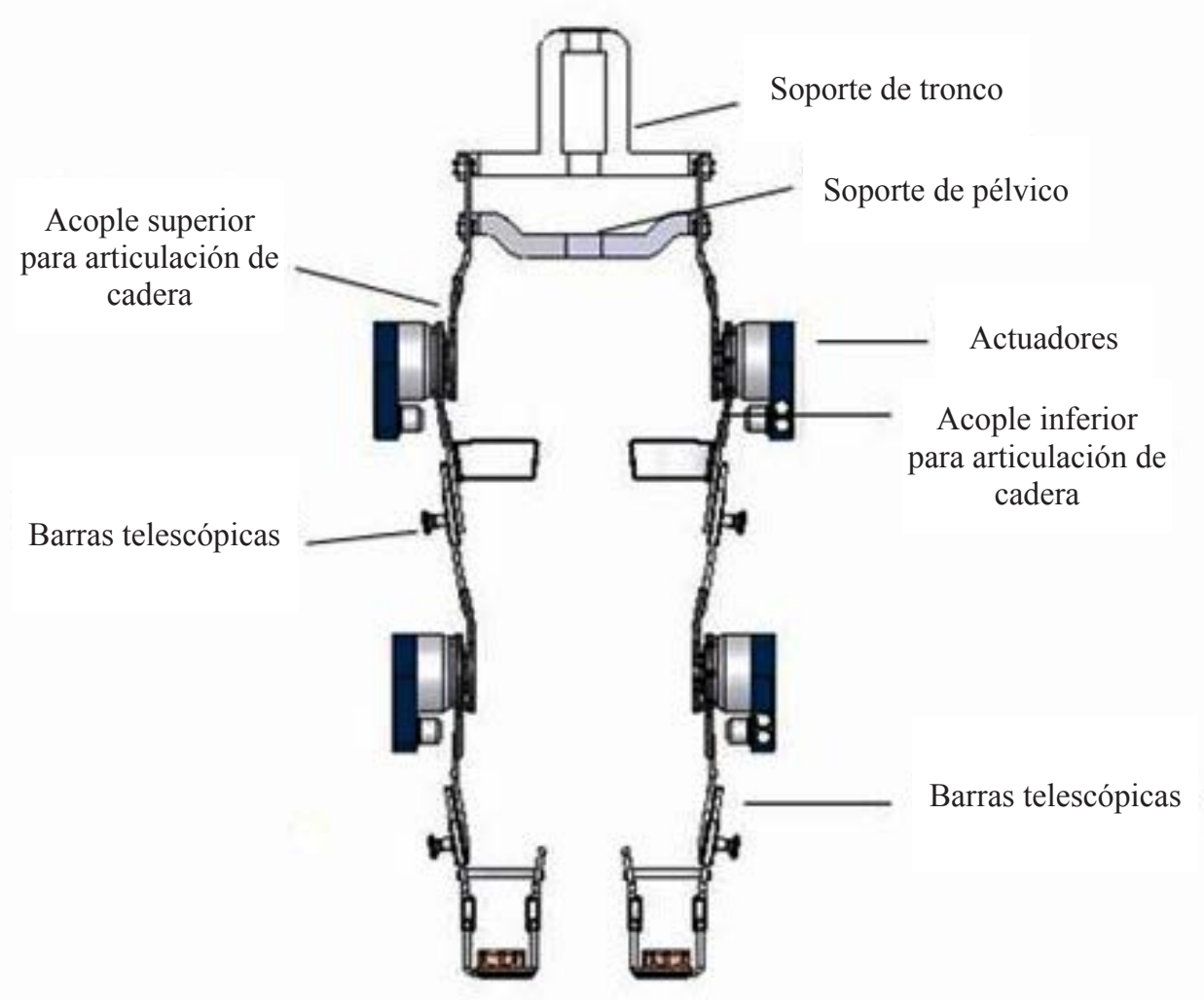

Fig. 2. Exoesqueleto de la Universidad del Valle para rehabilitación asistida de pacientes con pérdida parcial del movimiento de los miembros inferiores. 
Para el desarrollo de la interfaz se reunieron criterios que cubren aspectos técnicos, desde el campo de la ingeniería, y de otro lado, aspectos clínicos relacionados con el potencial de la herramienta tanto para pacientes como para terapeutas. Se desarrolló un trabajo interdisciplinar con un grupo de rehabilitadores de los principales centros de rehabilitación de la ciudad de Cali para determinar las principales necesidades buscando una herramienta de fácil manejo y gran utilidad. Dentro de los parámetros de retroalimentación que fueron considerados más pertinentes por los rehabilitadores están, los arcos de movilidad de cadera, rodilla y tobillo en el plano sagital, las señales electromiográficas, la distribución de presión sobre la planta del pie y la velocidad angular en cadera y rodilla. Este trabajo contó con el aval del comité de ética humana de la Universidad del Valle, Colombia, que determinó un estudio con riesgo mínimo. Se definieron todas las acciones para prevenir lesiones en los participantes durante las pruebas, así como también las acciones correctivas en caso de presentarse algún evento adverso. Cada participante recibió una explicación sobre su participación en el estudio y firmó un consentimiento informado antes de realizar las pruebas. Este trabajo contó con la aprobación del comité de ética de la Universidad del Valle, Colombia, para la realización de las pruebas de validación de la herramienta con el exoesqueleto.

Se estableció que el sistema debe permitir el seguimiento y evaluación del proceso de rehabilitación de las terapias. Además, permite la configuración y programación del sistema de instrumentación del exoesqueleto desde un $\mathrm{PC}$ de forma inalámbrica. Con esto, es posible determinar los sensores que pueden ser visualizados en la interfaz y configurar parámetros de ajuste para la calibración. La supervisión de la información de los sensores ubicados en la estructura mecánica y el paciente es realizada en modo On-line.Como sistemas de biofeedback, se estableció una realimentación visual y auditiva de acuerdo que permite al paciente para que participe activamente en las terapias y sea consciente de la evolución durante el proceso de rehabilitación, haciéndolo mucho más eficiente. Por último, los registros de cada terapia pueden ser guardados y consultados en el histórico de cada paciente.

\subsection{Biofeedback}

Para el biofeedback proporcionado por la interfaz, se emplearon los canales de sEMG obtenidos en ambos MMII y los sensores de la planta de los pies. Las señales sEMG fueron procesadas individualmente para cada canal, obteniendo una envolvente de la señal en el tiempo. Para la obtención de la envolvente, fue calculado el valor RMS de la señal sEMG en (1), usando el método de ventana deslizante de 200ms que separa conjuntos de muestras adyacentes para su procesamiento (Fig. 3). Este tiempo fue seleccionado de modo que el resultado pueda ser apreciado en tiempo real.

$$
r m s_{i}=\sqrt{\frac{1}{N} \sum_{k=0}^{N-1} x_{k}^{2}}
$$

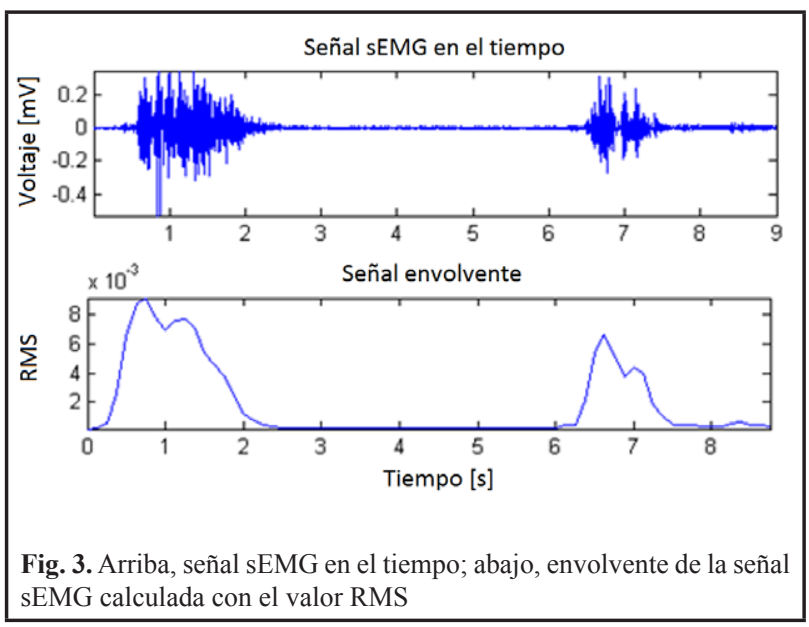

La señal envolvente obtenida se utiliza para tanto para el biofeedback visual como para el auditivo. En el primer caso, la señal es comparada con diferentes umbrales para determinar el rango de activación en el que se encuentra, usando escalas de colores que se relacionan proporcionalmente con el grado de activación muscular referente a la señal sEMG. En el segundo caso, la amplitud de la señal envolvente es relacionada con la frecuencia de una señal auditiva, igualmente de forma proporcional con la activación muscular.

Un esquema similar se utilizó con la señal de los sensores de presión ubicados en la planta de los pies, estableciendo una relación proporcional entre la amplitud de la señal y señal visual basada en tamaño y color.

\section{RESULTADOS}

La interfaz de usuario es concebida como una aplicación software, útil y de fácil manejo para el personal de salud, tomando en cuenta posibilidades tecnológicas, las necesidades de los pacientes, falencias de los procesos actuales en rehabilitación y posibles aportes del exoesqueleto en el reentrenamiento, evaluación y seguimiento de actividades motoras.

La herramienta software fue diseñada en JAVA con la ayuda de NetBeans IDE 6.8 y cuenta con las siguientes características:

- Permite el ingreso y consulta de registros de pacientes mediante una base de datos creada conMySQL server 5.0. 
- Comunicación con el sistema de procesamiento principal en el modo cliente servidor.

- Monitoreo y registro de variables del exoesqueleto obtenidas por la instrumentación.

- Estrategias de biofeedback.

La interfaz diseñada para el exoesqueleto cuenta con tres módulos: módulo 1, historia clínica del paciente; módulo 2, configuración, programación y mantenimiento; y módulo 3, monitoreo, registro y biofeedback. Un diagrama de bloques de la interfaz puede ser observado en la Fig. 4.

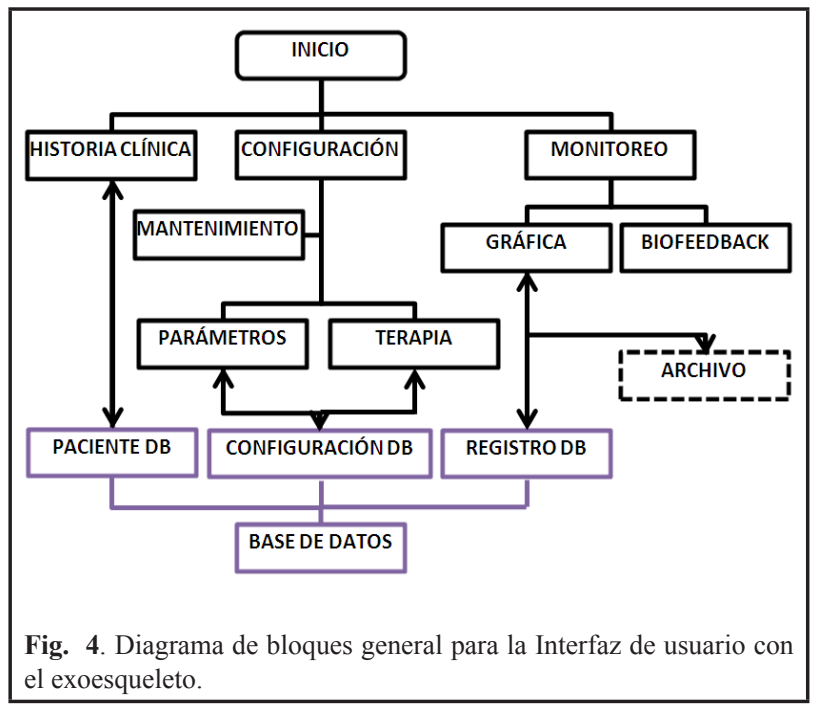

El módulo de historia clínica registra la información personal del paciente y permite el ingreso de algunos parámetros clínicos que determinan el tipo y nivel de lesión. El módulo de configuración, programación y monitoreo de tareas es uno de los más importantes para los pacientes y los terapeutas, dado que con él se pueden realizar la mayor cantidad de ajustes (Fig. 5). La principal función es la de proveer realimentación visual mediante el monitoreo de variables medidas a partir de la aplicación de técnicas de biofeedback (Fig. 6).

En el módulo de configuración del sistema se programan las terapias a realizar por parte del personal terapéutico, determinando parámetros de acuerdo al tipo de terapia y parámetros críticos. Estos parámetros definen la trayectoria a seguir por el actuador, el número de repeticiones, relación de asistencia y el tipo de control de bajo nivel a efectuar. Las trayectorias se establecen por parámetros como la posición angular inicial y final y se definen mediante una curva de "velocidad vs tiempo", en donde se determina la aceleración inicial del actuador, la velocidad a la que se mantendrá constante, y por último, la desaceleración hasta llegar nuevamente al reposo. Parámetros como ángulos críticos y velocidad máxima se establecen según el tipo de lesión y la patología del paciente para realizar una parada de emergencia del sistema completo.

Ya que se espera que el paciente incremente gradualmente su nivel de fuerza muscular a lo largo de su periodo de rehabilitación, se hace necesario ajustar el grado de asistencia proporcionado por el exoesqueleto mediante una valoración del nivel de fuerza muscular residual en el paciente a través del torque de la articulación de rodilla, para evitar que provea de una mayor asistencia de la necesaria que conlleve a prolongar la recuperación. Para esto se determina el torque necesario que debe ejercer el exoesqueleto para asistir únicamente su propio peso, obteniendo la relación de asistencia cero y posteriormente se incrementa gradualmente este parámetro hasta conseguir la correcta ejecución de la tarea propuesta.

Entre las actividades que se han concebido para realizar con el exoesqueleto se encuentran tareas de flexoextensión de rodilla, mantenimiento de la flexibilidad y del arco de movilidad articular (AMA), y el fortalecimiento y restablecimiento neuromuscular de la extremidad inferior afectada. Cada una de estas actividades que son determinadas por el terapeuta, son programadas a través de una secuencia de movimientos que determinan un conjunto de trayectorias a seguir por el sistema actuador.

La comunicación entre el exoesqueleto y la HMI es bidireccional. Los parámetros de configuración del exoesqueleto se programan en el nodo de procesamiento central desde la interfaz de usuario vía protocolo TCP. La adquisición de datos se configuró a $200 \mathrm{~Hz}$ y los datos son transmitidos al PC vía protocolo UDP. Un módulo de mantenimiento permite calibrar la escala de los valores de los sensores observados.

Para el monitoreo de la actividad paciente-exoesqueleto, se ha incluido la visualización on-line de algunas variables medidas en el exoesqueleto y de variables bioeléctricas en el paciente, que son convenientes y relevantes para el personal de la salud. La información que se visualiza podría ser útil para corregir los movimientos durante la terapia, evitando lesiones mayores o, por el contrario, proporcionar mayor libertad en los movimientos en los casos en que sea posible de acuerdo al progreso del paciente.

Dado que las señales sin procesar en el tiempo no serían fáciles de interpretar por un paciente, se determinó que el uso de otros esquemas basados en barras verticales podría evidenciar rápidamente la intensidad muscular con base en las sEMG procesadas. Además, se usaron colores que relacionan una baja intensidad con un color azul, hasta un color rojo que relaciona una intensidad fuerte. De igual forma, se incluyó un diagrama gráfico del cuerpo humano para señalar las zonas que están siendo medidas. Los colores y el tamaño de los círculos dibujados (Fig. 6) 
relacionan igualmente la intensidad muscular. De igual forma, se usaron las señales provenientes de los sensores de presión en los pies que para determinar la forma en que se ejerce la presión. Con eso se puede corregir el apoyo que realiza el paciente, que finalmente contribuye a la corrección del patrón de marcha.

Finalmente, se estableció un test de usabilidad que permite identificar la facilidad que puede tener un usuario durante su uso. Para esto, se realizó un proceso de evaluación en el cual se contó con la ayuda de un grupo de seis voluntarios, quienes estuvieron interesados en el sistema de apoyo a la rehabilitación desarrollado. En este proceso se contó igualmente con la participación de un personal técnico conocedor del sistema para la realimentación e identificación de mejoras en este aspecto.

Antes de la prueba de usabilidad se explicó al grupo de participantes sobre el manejo de la aplicación mediante una demostración del sistema completo. La prueba se basó en el "Test de Evaluación Heurística", que permite identificar mediante una encuesta realizada a los usuarios finales el nivel de satisfacción en el manejo en cuanto a los siguientes aspectos:

- Funcionalidad de la aplicación

- Calidad de la información relevante que se muestra y que se recibe

- Relación entre el sistema y el mundo real

- Control y libertad del usuario

- Consistencia y estándares

- Prevención de errores

- Capacidad de diagnóstico y manejo de errores

- Capacidad de dominio de la aplicación

- Estética y diseño

- Documentación

En la Tabla 1 se presenta el test realizado, donde se establece una calificación para cada aspecto de la evaluación, en el que 5 indica que el usuario está muy de acuerdo y 1 que está muy desacuerdo.
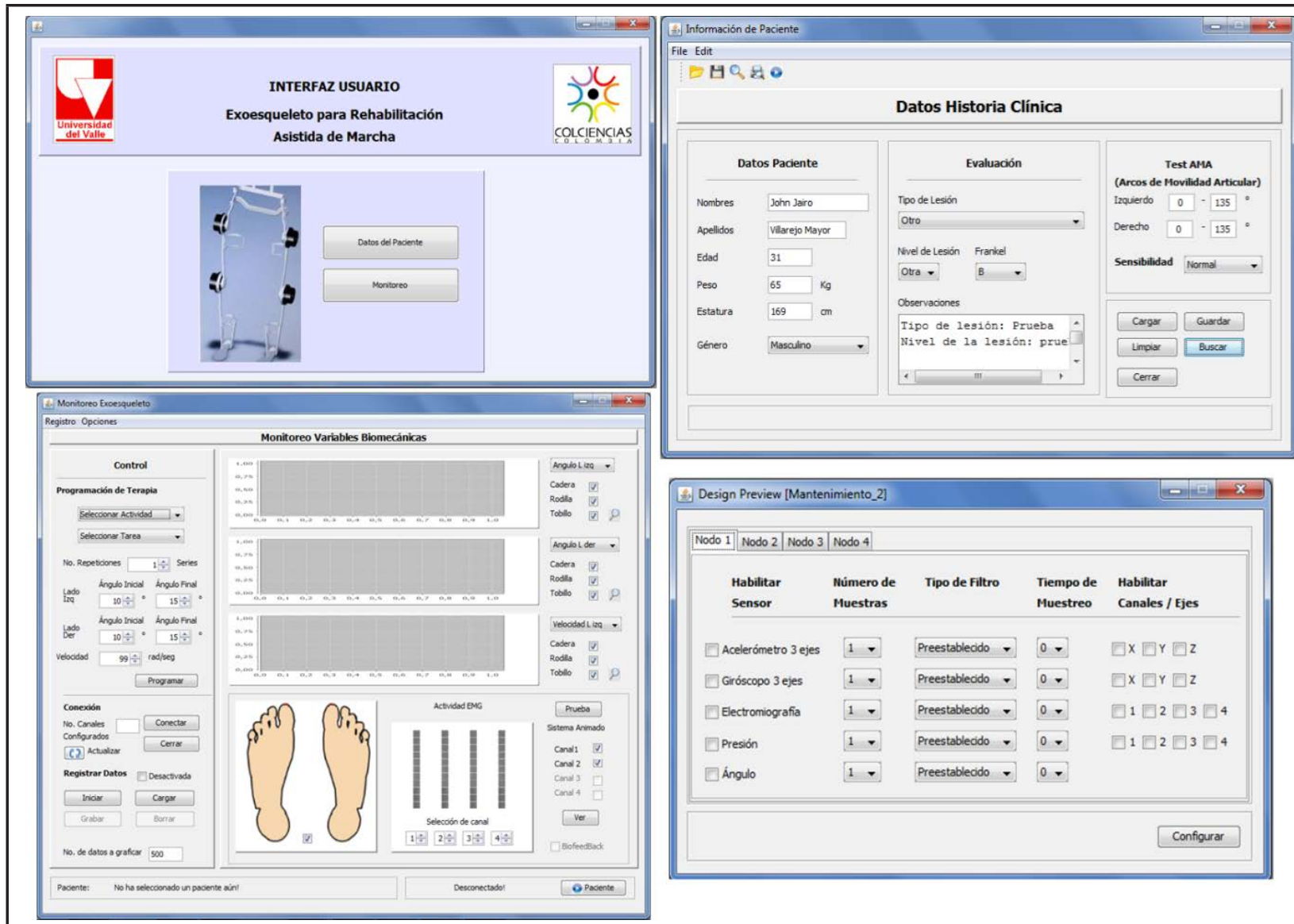

Fig. 5. Interfaz de Usuario del Exoesqueleto: (superior izquierda) Ventana de inicio; (superior derecha) Ventana de la Historia clínica; (inferior izquierda) Ventana de Configuración, Programación y Monitoreo de variables biomecánicas; (inferior derecha) Ventana de Mantenimiento 

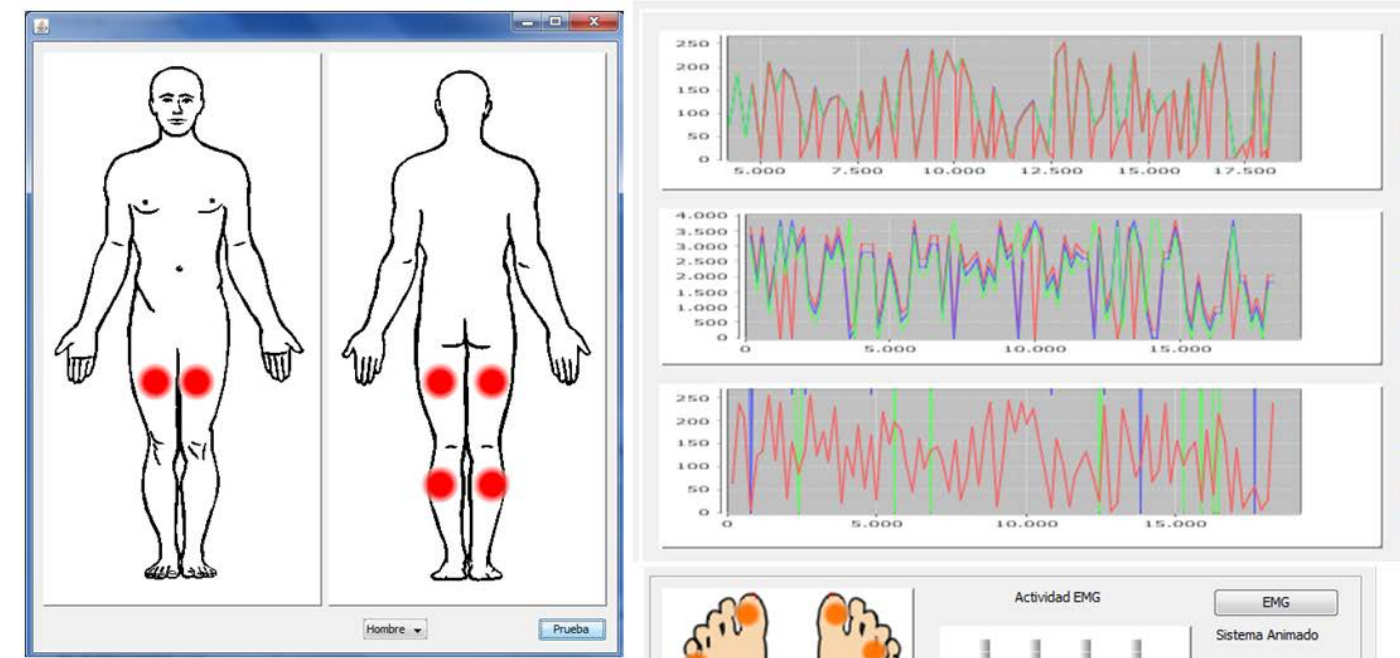

Angulo Lizq -

Cadera $\square$

Rodilla $\nabla$

Tobillo $\square \square$

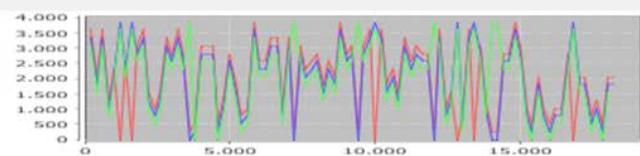

Angulo $L$ der $\quad$

Cadera

Rodilla $\square$

Tobillo $\nabla \rho$

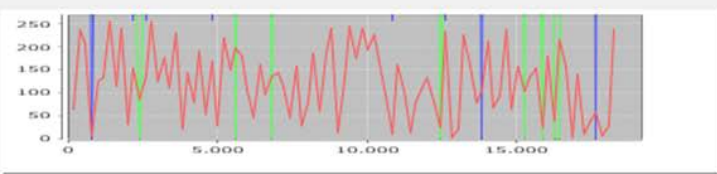

Velocidad Lizq -

Cadera $\square$

Rodilla $\square$

Tobillo $\nabla \rho$

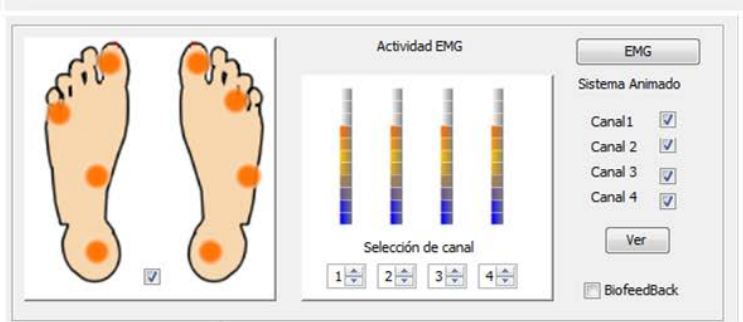

Fig. 6. Biofeedback realizado por la interfaz de usuario: (izquierda) Indicadores de actividad EMG; (superior derecha) Valores de ángulos y velocidad; (inferior derecha) Indicadores de presión y actividad EMG

Tabla 1. Resultados del Test de Usabilidad para la interfaz de usuario

\begin{tabular}{|c|c|}
\hline \multicolumn{2}{|l|}{ Visibilidad del estado del sistema } \\
\hline $\begin{array}{l}\text { La aplicación muestra claramente dónde se encuentra el } \\
\text { usuario }\end{array}$ & 4 \\
\hline $\begin{array}{l}\text { Los accesos posibles de explorar en la aplicación están } \\
\text { claramente señalados e identificados }\end{array}$ & 5 \\
\hline \multicolumn{2}{|l|}{ Relación entre sistema y el mundo Real } \\
\hline El lenguaje es claro & 4 \\
\hline Los conceptos utilizados son entendibles & 4 \\
\hline Las palabras son de significado conocido & 4 \\
\hline Los iconos generan significado & 4 \\
\hline \multicolumn{2}{|l|}{ Control del Usuario y Libertad } \\
\hline Es fácil regresar a las interfaces ya exploradas & 5 \\
\hline Es fácil volver a la interface principal & 4 \\
\hline Existen botones para dar paso a otra interface & 5 \\
\hline \multicolumn{2}{|l|}{ Consistencia y Estándares } \\
\hline $\begin{array}{l}\text { Existe coherencia entre el nombre del botón y la interface } \\
\text { al que apunta }\end{array}$ & 4 \\
\hline Todos los botones tienen contenido & 5 \\
\hline $\begin{array}{l}\text { Existe coherencia entre el título de la interface y su } \\
\text { contenido }\end{array}$ & 5 \\
\hline Solo existe un solo botón que lleve a una misma interface & 5 \\
\hline \multicolumn{2}{|l|}{ Prevención de Errores } \\
\hline Existen mensajes que prevengan posibles errores & 4 \\
\hline Es posible prever posibles errores & 4 \\
\hline
\end{tabular}

\begin{tabular}{|c|c|}
\hline La aplicación no induce a cometer errores & 4 \\
\hline \multicolumn{2}{|l|}{ Reconocer en Lugar de Recordar } \\
\hline Los iconos son fácilmente reconocibles & 4 \\
\hline Los botones pueden identificarse claramente & 4 \\
\hline Es posible reconocer dónde se encuentra el usuario & 4 \\
\hline \multicolumn{2}{|l|}{ Estética y Diseño Minimalista } \\
\hline La información es relevante & 4 \\
\hline El contenido está bien clasificado & 3 \\
\hline El contenido está correctamente organizado & 4 \\
\hline El contenido está bien distribuido en la aplicación & 4 \\
\hline \multicolumn{2}{|l|}{ Reconocimiento, Diagnostico y Recuperación de Errores } \\
\hline Es fácil reconocer cuando ocurre un error & 4 \\
\hline $\begin{array}{l}\text { Después que ocurre un error es fácil volver a la interface } \\
\text { de origen }\end{array}$ & 5 \\
\hline $\begin{array}{l}\text { Cuando ocurre un error existen mecanismos para } \\
\text { solucionarlos }\end{array}$ & 4 \\
\hline \multicolumn{2}{|l|}{ Ayuda y Documentación } \\
\hline $\begin{array}{l}\text { Existe algún tipo de ayuda o indicación de soporte en la } \\
\text { aplicación }\end{array}$ & 3 \\
\hline Cuando existe ayuda, ésta es específica & 2 \\
\hline La ayuda está asequible & 4 \\
\hline
\end{tabular}

Los resultados de la evaluación indicaron que la aplicación resulta de gran interés para los terapeutas y se consideró como una herramienta importante para el acompañamiento de las terapias. 


\section{Conclusiones}

Una aplicación software para la interacción con un dispositivo exoesquelético concebido para rehabilitación física representa una herramienta de apoyo para terapeutas y médicos especializados, que intervengan en el proceso de recuperación de pacientes que conserven algún tipo de movilidad en sus músculos para su restauración. El paciente es el principal beneficiado del sistema, puesto que el principal propósito es el de facilitar y reducir el tiempo empleado durante el proceso. Con esta herramienta se integran diferentes métodos para realizar biofeedback actuando en conjunto con otras variables biomecánicas para permitir al terapeuta realizar análisis más detallados. Esta realimentación brinda una información complementaria para el paciente que le permite conocer su desempeño y exigirse más en los entrenamientos para su beneficio. Este estudio de carácter multidisciplinar implica la integración de diversas áreas de conocimiento y la apropiación de conceptos y comprensión de técnicas de campos diferentes al de la ingeniería, por lo cual representa un aporte considerable para futuros proyectos relacionados con el desarrollo de ayudas técnicas en apoyo a la discapacidad, desde el punto de vista de la concepción y el planteamiento de soluciones para problemas de la salud. El software desarrollado fue registrado ante la Dirección Nacional de Derechos de Autor, Ministerio del Interior, Colombia, con el número de registro: 1-2012-22057.

\section{Agradecimientos}

Este estudio fue apoyado por la Universidad del Valle, Colombia, a través de la financiación del proyecto CI1679, de la convocatoria interna, año 2011. También, se contó con el apoyo de Colciencias a través de la financiación del proyecto 1106-452-21163, año 2009. Finalmente, los autores agradecen al Grupo de Investigación en Control Industrial, GICI, de la Universidad del Valle y al profesor José Miguel Ramírez Scarpetta por su orientación en el proyecto.

\section{REFERENCIAS}

[1]. Carballo Pérez, E.S. (2015), "Estudio de la amplitud del movimiento articular del hombro en paciente Hemiplejicos sometidos a un programa de Neurorrehabilitacion intensiva. Estudio preliminar", available at: http://www.ilustrados.com/ publicaciones/EEyFplEpAFOGcMjiLH.php (accessed 3 May 2015).

[2]. Broome, R.T. (2005), Técnica Quiropráctica de las Articulaciones Periféricas, (Broome, R.T.,Ed.), Paidotribo.

[3]. Hunt, K.J. (2005), Control systems for function restoration, exercise, fitness and health in spinal cord injury, Doctoral Thesis, University of Glasgow.
[4]. Schmidt, R.A. and Lee, T.D. (2011), Motor Control and Learning, A Behavioral Emphasis, Human Kinetic, 5th Ed.

[5]. Shumway-Cook, A. and Woollacott, M.H. (2007), Motor Control: Translating Research Into Clinical Practice, Lippincott Williams \& Wilkins, Philadelphia, Pennsylvania, 3rd Ed.

[6]. Dobkin, B.H. (2005), "Rehabilitation and functional neuroimaging dose-response trajectories for clinical trials.", Neurorehabilitation and neural repair, United States, Vol. 19 No. 4, pp. 276-282.

[7]. Kwakkel, G., Van Peppen, R., Wagenaar, R.C., Dauphinee, S.W., Richards, C., Ashburn, A., Miller, K., et al. (2004), "Effects of augmented exercise therapy time after stroke: A meta-analysis", Stroke, Vol. 35 No. 11, pp. 2529-2536.

[8]. Kwakkel, G. and Wagenaar, R.C. (2002), "Effect of duration of upper- and lower-extremity rehabilitation sessions and walking speed on recovery of interlimb coordination in hemiplegic gait.", Physical therapy, Vol. 82 No. 5, pp. 432-448.

[9]. Wang, D., Lee, K.-M., Guo, J. and Yang, C.-J. (2014), “Adaptive Knee Joint Exoskeleton Based on Biological Geometries", Mechatronics, IEEE/ASME Transactions on.

[10]. Pietrusinski, M., Cajigas, I., Severini, G., Bonato, P. and Mavroidis, C. (2014), "Robotic Gait Rehabilitation Trainer", Mechatronics, IEEE/ASME Transactions on.

[11]. Wang, S., Wang, L., Meijneke, C., van Asseldonk, E., Hoellinger, T., Cheron, G., Ivanenko, Y., et al. (2015), "Design and Control of the MINDWALKER Exoskeleton.”, IEEE transactions on neural systems and rehabilitation engineering : a publication of the IEEE Engineering in Medicine and Biology Society, United States, Vol. 23 No. 2, pp. 277-286.

[12]. Lu, R., Li, Z., Su, C.-Y. and Xue, A. (2014), "Development and Learning Control of a Human Limb With a Rehabilitation Exoskeleton", Industrial Electronics, IEEE Transactions on.

[13]. Basmajian, J. V. (1978), Muscles Alive-their functions revealed by electromyography, Williams \& Wilkins, Baltimore, 4th Ed.

[14]. Lünenburger, L., Colombo, G. and Riener, R. (2007), "Biofeedback for robotic gait rehabilitation.", Journal of neuroengineering and rehabilitation, Vol. 4, p. 1.

[15]. Schmidt, R.A. and Lee, T.D. (2014), Motor Learning and Performance, Human Kinetic, 5th Ed.

[16]. Krakauer, J.W. (2006), "Motor learning: its relevance to stroke recovery and neurorehabilitation.", Current opinion in neurology, Vol. 19 No. 1, pp. 84-90.

[17]. Cook, A.M. and Polgar, J.M. (2008), Cook and Hussey's Assistive Technologies: Principles and Practice, Mosby Elsevier, 4th Ed.

[18]. Rodríguez-Hernández, A.F., Merino, C., Casanova, O., Modrono, C., Torres, M.Á., Montserrat, R., Navarrete, G., et al. (2010), "Sensory substitution for visually disabled people: Computer solutions", WSEAS Transactions on Biology and Biomedicine, Vol. 7 No. 1, pp. 1-10.

[19]. Morasso, P. (2013), "Towards an Integrated Approach to Multimodal Assistance of Stroke Patients Based on the Promotion of Intentionality", in Pons, J.L., Torricelli, D. and Pajaro, M. (Eds.),Converging Clinical and Engineering Research on Neurorehabilitation, Biosystems and Biorobotics, Springer, pp. 23-27.

[20]. Sharma, R., Pavlovic, V.I. and Huang, T.S. (1998), "Toward Multimodal Human-Computer Interface", Proceedings of the IEEE, Vol. 86 No. 5, pp. 853-869. 
[21]. Teasell, R.W., Bhogal, S.K., Foley, N.C. and Speechley, M.R. (2003), "Gait retraining post stroke.", Topics in stroke rehabilitation, Vol. 10 No. 2, pp. 34-65.

[22]. Goldfarb, M. and Durfee, W.K. (1996), "Design of a controlled brake orthosis for FES-aided gait", IEEE Trans Rehabil Eng, Vol. 4 No. 1, pp. 13-24.

[23]. Quiñones, I., Vela, E., Pérez, a. I., Alessi, a., Urrusti, J.L. and Cepeda, D. (2009), "Biofeedback system for transfemoral amputees rehabilitation", 2009 Pan American Health Care Exchanges - PAHCE 2009, pp. 148-152.

[24]. Wolf, S.L. (1983), "Electromyographic biofeedback applications to stroke patients. A critical review.", Physical therapy, Vol. 63 No. 9, pp. $1448-1459$.

[25]. Moreland, J.D., Thomson, M.A. and Fuoco, A.R. (1998), "Electromyographic biofeedback to improve lower extremity function after stroke: A meta-analysis", Archives of Physical Medicine and Rehabilitation, Vol. 79 No. 2, pp. 134-140.

[26]. López Beltrán, E. (2004), "Electrogoniómetro como dispositivo de realimentación (biofeedback) para electro-estimulación controlada", Revista de Ciencia, Tecnología y Medio Ambiente, Vol. II.

[27]. Chu Kazerooni, H., Zoss, A., A. (2005), "On the Biomimetic Design of the Berkley Lower Extremity Exoskeleton (BLEEX)", Proceedings of the IEEE, No. April, pp. 4345-4352.

[28]. Chevallereau, B.C., Abba, G., Aoustin, Y., Plestan, F., Westervelt, E.R., Canudas-de-wit, C. and Grizzle, J.W. (2003), "RABBIT: A Testbed for Advanced Control Theory", IEEE Control Systems Mag., Vol. 23 No. 5, pp. 57-79.

[29]. Giggins, O.M., Persson, U.M. and Caulfield, B. (2013), "Biofeedback in rehabilitation.", Journal of neuroengineering and rehabilitation, Vol. 10 No. 1, p. 60.

[30]. Krasny-Pacini, A., Hiebel, J., Pauly, F., Godon, S. and Chevignard, M. (2013), "Goal attainment scaling in rehabilitation: a literature-based update.", Annals of physical and rehabilitation medicine, Netherlands, Vol. 56 No. 3, pp. 212-230.

[31]. Boudarham, J., Roche, N., Teixeira, M., Hameau, S., Robertson, J., Bensmail, D. and Zory, R. (2014), "Relationship between neuromuscular fatigue and spasticity in chronic stroke patients: a pilot study.", Journal of electromyography and kinesiology: official journal of the International Society of Electrophysiological Kinesiology, England, Vol. 24 No. 2, pp. 292-299.

[32]. Chávez Cardona, M.A., Rodríguez Spitia, F. and Barandica López, A. (2010), "Exoesqueletos para potenciar las capacidades humanas y apoyar la rehabilitación", Revista ingeniería Biomédica, Vol. 4, pp. 63-73.

[33]. Villarejo Mayor, J.J. and Caicedo Bravo, E.F. (2012), Detección de la Intención de Movimiento en la Interfaz Hombre-Máquina de un Exoesqueleto para Rehabilitación, Masters Thesis, Master of Electronic Engineering, Universidad del Valle. 